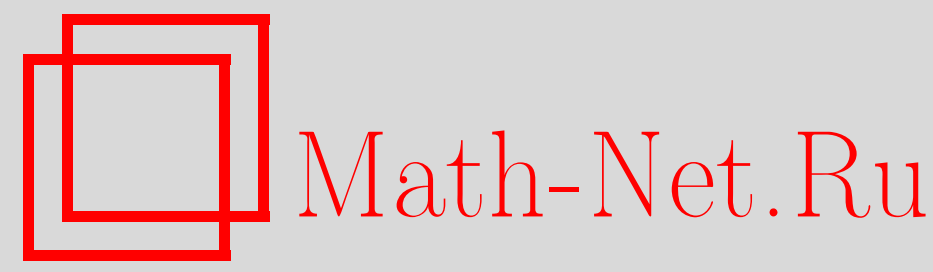

Е. Г. Скляренко, О гомологиях окружения, Матем. сб., 1997, том 188, номер 2, 129 136

DOI: https://doi.org/10.4213/sm204

Использование Общероссийского математического портала Math-Net.Ru подразумевает, что вы прочитали и согласны с пользовательским соглашением

http: //www . mathnet.ru/rus/agreement

Параметры загрузки:

IP : 35.173 .219 .149

26 апреля 2023 г., 18:06:51 
УДК 515.142.21

\title{
Е.Г. Скляренко
}

\section{О гомологиях окружения}

\begin{abstract}
Показывается, что гомологии окружения подпространства, являющегося в объемлющем пространстве слабым окрестностным деформационньм ретрактом, со сдвигом в размерности на +1 изоморфны гомологиям связи этого подпространства с его дополнением.

Библиографоия: 8 названий.
\end{abstract}

Гомологии множества $W \backslash L$, где $W$ - регулярная окрестность подкомплекса $L$ симплициального комплекса $K$, не зависят от выбора $W$, поэтому их естественно называть гомологиями окружения $L$ в $K$. Для менњшей регулярной окрестности $W^{\prime} \subset W$ имеем

$H_{p}(K, L \cup(K \backslash \operatorname{Int} W))=H_{p}\left(K, W^{\prime} \cup(K \backslash \operatorname{Int} W)\right)=H_{p}\left(W \backslash \operatorname{Int} W^{\prime}, \partial W \cup \partial W^{\prime}\right)$.

Поскольку $W \backslash \operatorname{Int} W^{\prime}$ - произведение границы $\partial W$ на сегмент, а $W \backslash L$ стягивается на $\partial W$, то

$H_{p+1}(K, L \cup(K \backslash \operatorname{Int} W))=H_{p+1}(\partial W \times I, \partial W \times\{0\} \cup \partial W \times\{1\})=H_{p}(W \backslash L)$,

т.е. гомологии окружения совпадают при сдвиге на единицу размерности с гомологиями связи подкомплекса с его дополнением.

Это наблюдение перестает быть тривиальным сразу за пределами категории полиэдров. Пусть $A$ и $B=X \backslash A$ - дополнительные подпространства пространства $X, P$ и $Q$ - замкнутые в $X$ подпространства, причем $P \subset A$ и $Q \subset B$. Визуально не обнаруживается никакой связи между гомологиями пары $(X, P \cup Q)$ и множества $X \backslash(P \cup Q)$, в том числе и когда $A$, например, замкнуто (в этом случае естественно полагать $P=A$ ). Естественно понимать под гомологиями связи $H_{p}^{\varphi}(A: B ; \mathscr{G})$ в $X$ между дополнительньпи подпространствами $A$ и $B$ прямой предел групп $H_{p}^{\varphi}(X, P \cup Q ; \mathscr{G})$ по всем указанным $P$ и $Q$. Естественность этого определения подтверждается, в частности, тем, что

$$
H_{p}^{\varphi}(A ; \mathscr{G})=\underset{P \subset A}{\lim _{P \subset A}}\left\{H_{p}^{\varphi}(P ; \mathscr{G})\right\}
$$

и

$$
H_{p}^{\varphi}(B ; \mathscr{G})=\underset{Q \subset B}{\lim _{Q}}\left\{H_{p}^{\varphi}(Q ; \mathscr{G})\right\}
$$

Работа выполнена при финансовой поддержке Российского фонда фундаментальных исследований (грант № 97-01-00174). 
Здесь $\varphi$ - некоторое семейство носителей, $\mathscr{G}$ - локально постоянные коэффициенты.

Возможен и другой способ изучения связей между $A$ и $B$. Пусть $V=X \backslash P$, $W=X \backslash Q$ и $U=W \cap V=X \backslash(P \cup Q)$. Фактически $W$ и $V$ - всевозможные окрестности $A$ и $B$ в $X$. Гомологические связи между $A$ и $B$ отражают, очевидно, обратные пределы по $U$ групп $H_{p}^{\varphi}(U ; \mathscr{G})$. Однако, с учетом неточности функтора обратного предела $\lim$ (в отличие от $\lim$ ) естественнее рассматривать гипергомологии (в смысле [1]) для функтора $F=\lim$ проективной системы $\left\{C_{*}^{\varphi}(U ; \mathscr{G})\right\}$ определяющих нужные гомологии цепных комплексов, которая рассматривается как цепной комплекс в категории проективных систем абелевых групп (или модулей). Ясно, что это нетривиальный комплекс (несмотря на нулевое пересечение $\left.\bigcap_{U} C_{*}^{\varphi}(U ; \mathscr{G})\right)$. Указанные гипергомологии будем обозначать через $H_{p}^{\varphi}(A \mid B ; \mathscr{G})$ и назьвать гомологиями контакта между $A$ и $B$ в $X$. Групшы $\lim _{U}\left\{H_{p}^{\varphi}(U ; \mathscr{G})\right\}$ тесно связаны с ними, связь осуществляется через одну из отвечающих гипергомологиям стандартных спектральных последовательностей.

Гомологии связи и контакта подробно изучались в [7], где они включались в разного рода точные гомологические последовательности и составленные такими последовательностями диаграммы. Установлено наличие естественных преобразований $\beta: H_{p}^{\varphi}(A: B ; \mathscr{G}) \rightarrow H_{p-1}^{\varphi}(A \mid B ; \mathscr{G})$. Тщательно анализировались общие условия изоморфизма.

Интерес представляет прежде всего тот случай, когда одно из подпространств, пусть это будет $A$, замкнуто. В этом случае

$$
H_{p}^{\varphi}(A: B ; \mathscr{G})=\underset{W}{\lim }\left\{H_{p}^{\varphi}(X, A \cup(X \backslash W) ; \mathscr{G})\right\}
$$

а группы $H_{p}^{\varphi}(A \mid B ; \mathscr{G})$ - суть гипергомологии для функтора $\lim ^{\longleftarrow}$ комплекса $\left\{C_{*}^{\varphi}(W \backslash A ; \mathscr{G})\right\}$. Будем обозначать последние через $\left.H_{p}^{\varphi}(\langle A\rangle\rangle ; \overleftarrow{\mathscr{G}}\right)$ и назьвать гомологиями окружсения $A$ в $X$. В работе [7] установлено, что в случае компактного $A$ преобразования $\beta$ изоморфны, если либо $X$ локально компактно, либо $X$ паракомпактно, но $A$ имеет в $X$ счетную фундаментальную систему окрестностей. Автор не располагает какими-либо примерами неизоморфности $\beta$ (в том числе и для произвольных $A$ и $B$ ). Вопрос об изоморфности $\beta$ для некомпактных замкнутых множеств уже в случае классического семейства $\varphi=c$ компактных множеств, в том числе в локально компактных пространствах, оказывается неожиданно сложным: для сравнительно простых подмножеств евклидовой плоскости он, вообше говоря, неразрешим в системе аксиом Цермело-Френкеля с аксиомой выбора $[7, \S 6]$.

Как это нередко бывает, когомологический вариант проблемы оказывается проще: для обычных когомологий ( $\varphi$ - семейство всех замкнутых множеств) соответствующие преобразования изоморфын для любых замкнутых подпространств паракомпактных хаусдорфовых пространств, в том числе для любых пучков коэффициентов $[7, \S 7]$. Гомологии (и когомологии) связи и окружения представляют интерес и в том частном случае, когда $A=x$ - одна точка, обеспечивая единый общий подход к теории локальных гомологий (и когомологий) $[7, \S 8]$.

В настоящей работе изоморфность $\beta$ доказывается в случае, когда $A$ - замкнутое подпространство, являющееся слабьм окрестностным деформационным ретрактом содержащего его паракомпактного (даже просто нормального) хаусдорфова пространства $X$ (для любой окрестности $W$ подпространства $A$ найдется 
меньшая, стягиваемая в $A$ по $W$ таким образом, что в процессе всего стягивания образ $A$ остается в $A$ ). Семейство носителей $\varphi$ предполагается таким, что в процессе рассматриваемых деформаций образы множеств из $\varphi$ остаются в $\varphi$. Это всегда так, например, для классического семейства $\varphi=c$. Вложение $A \subset W$ и результат стягивания $W$ в $A$ обеспечивают соотношения

$$
H_{n}^{\varphi}(W ; \mathscr{G})=H_{n}^{\varphi}(A ; \mathscr{G}) \oplus H(W)_{n} .
$$

При $W^{\prime} \subset W$ слагаемое $H_{n}^{\varphi}(A ; \mathscr{G})$ в этих группах отображается тождественно, а для окрестности $W^{\prime}$, стягивающейся в $A$ по $W$, образ $H\left(W^{\prime}\right)_{n}$ в $H_{n}^{\varphi}(W ; \mathscr{G})$ равен нулю. Таким образом, проективная система групп $\left\{H_{n}^{\varphi}(W ; \mathscr{G})\right\}$ содержит постоянную подсистему $\left\{H_{n}^{\varphi}(A ; \mathscr{G})\right\}$, для которой факторсистема $\left\{H_{n}^{\varphi}(W ; \mathscr{G}) / H_{n}^{\varphi}(A ; \mathscr{G})\right\}$ тривиальна (т.е. с нулевыми проекциями для пар $W^{\prime} \subset W$ с достаточно малыми относительно $W$ окрестностями $W^{\prime}$ подпространства $A$ ). Будем называть такие проективные системы почти постоянными.

Напомним, что на любой постоянной системе функторы $\lim ^{p}$ обращаются в нуль при $p>0$ (хотя бы в силу теоремы 1.8 из [3]), а на любой тривиальной системе $\lim ^{p}=0$ при всех $p[4$, лемма 5]. Из точной последовательности функторов

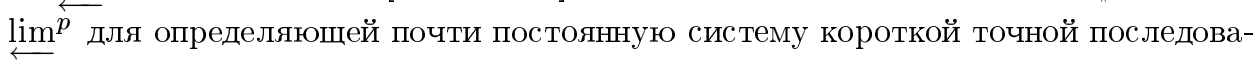

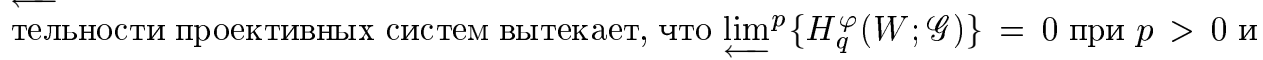
$\lim _{\longleftarrow}\left\{H_{q}^{\varphi}(W ; \mathscr{G})\right\}=H_{q}^{\varphi}(A ; \mathscr{G})$.

Обсуждаемьй результат вытекает из следуюшего чуть более общего утверждения.

ТЕОРема 1. Пусть $A$ - замкнутое подпространство нормального пространства $X$, для достаточно малых окрестностей $W$ которого вложения $A \subset W$ индуцируют мономорфизмы $H_{n}^{\varphi}(A ; \mathscr{G}) \subset H_{n}^{\varphi}(W ; \mathscr{G})$, превращающие проективные системы групп $\left\{H_{n}^{\varphi}(W ; \mathscr{G})\right\}$ в почти постоянные. При этом условии преобразования

$$
\beta: H_{n}^{\varphi}(A: X \backslash A ; \mathscr{G}) \rightarrow H_{n-1}^{\varphi}(\langle\langle A\rangle\rangle ; \mathscr{G})
$$

- изоморфизмыл.

Несколько более слабая версия этой теоремы была представлена в [7, 3$]$. Намеченное там короткое доказательство апеллировало к известной работе Эйленберга и Мура о пределах и спектральных последовательностях и опиралось на сходимость "второй" спектральной последовательности (отвечающей неограниченной снизу фильтрации) к "сильным" гомологиям двойного комплекса, полная градуировка в котором определялась не прямыми суммами по $p+q=$ const (как это обычно), а прямыми произведениями однородных составляющих бистепеней $(p, q)$, что обеспечивало его полноту (в смысле Эйленберга и Мура) по отношению к рассматриваемой фильтрации. Поскольку условия сходимости не были выделены явно (a, как показывает пример g) из $[8, \S 3]$, ср. там же с теоремой 1.4 и следствием 1.5, такие условия в общих ситуациях очень существенны), использованная сходимость в конечном итоге скорее оказывалась следствием результата, чем основой его доказательства (в доказательстве фактически возник пробел). В соответствии с намеченной в [7] схемой рассуждений не оптимальным образом выбраны 
и используемые там вспомогательные фильтрующие подкомплексы “первой” спектральной последовательности.

Теорема доказывается ниже без апелляции к "сильным" гомологиям. Используется практически та же идея, что и в [7], но вторая спектральная последовательность анализируется применительно к обычным гомологиям двойного комплекса, определяющим исследуемые гипергомологии. Подтверждается в конечном итоге и ее сходимость к сильным гомологиям, более того, они оказываются изоморфны обычным. По сравнению с [7] усилено понятие почти постоянной проективной системы. Основная часть доказательства сводится к проверке некоторого общего гомолого-алгебраического утверждения, связанного с взаимозависимостью двух спектральных последовательностей, которое формулируется и проверяется в тер-

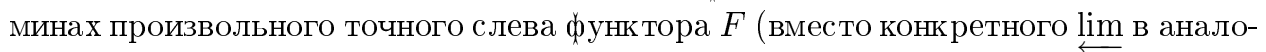
гичных ситуация в [7]). Из теоремы следует

ПРЕДЛОЖЕНИЕ 2. В условиях теоремы 1 проективные системы $\left\{H_{n}^{\varphi}(W \backslash A ; \mathscr{G})\right\}$ почти постоянны, а их постоянные составляющие изоморфнь гомологиям окружения $H_{n}^{\varphi}(\langle\langle A\rangle ; \mathscr{G})$.

Доказательство дано в $[7, \S 3]$.

В силу следствия 2.3 из [7] $\beta$ тогда и только тогда изоморфизмы, когда гипергомологии для функтора $F=\longleftarrow \lim$ проективной системы $\left\{C_{*}^{\varphi}(W ; \mathscr{G})\right\}$ (как цепного комплекса) совпадают с $H_{*}^{\varphi}(A ; \mathscr{G})$. Докажем это совпадение. Обозначим указанные гипергомологии $\left\{C_{*}^{\varphi}(W ; \mathscr{G})\right\}$ через $H_{*}^{\varphi}(A \subset X ; \mathscr{G})$. Они определяются как гомологии двойного комплекса в полной градуировке, расположенного в “верхней полуплоскости" и получающегося после применения функтора lim к любой lim-ацикличной резольвенте в смысле главы XVII в [1] комплекса $\left\{C_{*}^{\varphi}(W ; \mathscr{G})\right\}$ (например, составленной известньми резольвентами Рооса входящих в $\left\{C_{*}^{\varphi}(W ; \mathscr{G})\right\}$ проективных систем и оказьваюшейся резольвентой Картана-Эйленберга для $\left\{C_{*}^{\varphi}(W ; \mathscr{G})\right\}$ в силу функториальности резольвенты Рооса). Поскольку фильтрация по $p$ регулярна, к гипергомологиям сходится “первая" (в терминологии главы XVII в [1]) спектральная последовательность со вторым членом вида

$$
{ }^{\prime} E_{2}^{p q}=H_{-p}\left(\lim ^{q}\left\{C_{*}^{\varphi}(W ; \mathscr{G})\right\}\right)
$$

$\left({ }^{\prime} E_{2}^{p q}=0\right.$ при $\left.p>0\right)$. Поскольку

$$
\lim _{\longleftarrow}\left\{C_{*}^{\varphi}(W ; \mathscr{G})\right\}=\bigcap_{W} C_{*}^{\varphi}(W ; \mathscr{G})=C_{*}^{\varphi}(A ; \mathscr{G}),
$$

имеем ' $E_{2}^{p 0}=H_{-p}^{\varphi}(A ; \mathscr{G})$. Теорема будет доказана, если окажется, что ' $E_{2}^{p q}=0$ при $q>0$ (в этом случае $\left.H_{-p}^{\varphi}(A \subset X ; \mathscr{G})={ }^{\prime} E_{2}^{p 0}\right)$.

Для второй спектральной последовательности имеем (сохраняя прежний смысл индексов $p$ и $q$ )

$$
{ }^{\prime \prime} E_{2}^{q p}=\lim ^{q}\left\{H_{-p}^{\varphi}(W ; \mathscr{G})\right\} .
$$

В соответствии со сделанньми выше наблюдениями " $E_{2}^{q p}=0$ при $q>0$ и ${ }^{\prime \prime} E_{2}^{0 p}=$ $H_{-p}^{\varphi}(A ; \mathscr{G})$. В частности, имеем ${ }^{\prime} E_{2}^{p 0}={ }^{\prime \prime} E_{2}^{0 p}$. Поскольку фильтрация по $q$ не 
регулярна, не выяснен пока вопрос о сходимости второй спектральной последовательности к нужньм гипергомологиям (которая, если и сходится, то обычно к упоминавшимся выше "сильньм" гомологиям).

Возникаюшая задача становится более прозрачной при рассмотрении вместо конкретного функтора $\lim$ любого точного слева аддитивного функтора $F$. Пусть $\mathscr{C}^{*}=\left\{\mathscr{C}^{q},-\infty<q \longleftarrow \infty\right\}-$ коцепной комплекс (в случае цепного полагаем $\left.\mathscr{C}^{q}=\mathscr{C}_{-q}\right), \mathscr{J}^{*} \mathscr{C}^{*}=\bigoplus_{p, q} \mathscr{J}^{p} \mathscr{C}^{q}$-некоторая $F$-ацикличная (не обязательно инъективная) резольвента Картана-Эйленберга [1, гл. XVII] этого комплекса. Гиперкогомологии $\mathbb{H}^{*}\left(\mathscr{C}^{*}\right)$ комплекса $\mathscr{C}^{*}$ для функтора $F$ совпадают с когомологиями рассматриваемого в полной градуировке $p+q=n$ комплекса $F\left(\mathscr{J}^{*} \mathscr{C}^{*}\right)$ (см., например, [8, предложение 2.2]). Имеем

$$
{ }^{\prime} E_{2}^{q p}=H^{q} F^{p}\left(\mathscr{C}^{*}\right), \quad{ }^{\prime \prime} E_{2}^{p q}=F^{p} H^{q}\left(\mathscr{C}^{*}\right) .
$$

В частности,

$$
{ }^{\prime} E_{2}^{q 0}=H^{q} F\left(\mathscr{C}^{*}\right), \quad{ }^{\prime \prime} E_{2}^{0 q}=F H^{q}\left(\mathscr{C}^{*}\right) .
$$

Лемма 3. Имеется естественное преобразование $\alpha:{ }^{\prime} E_{2}^{q 0} \rightarrow{ }^{\prime \prime} E_{2}^{0 q}$.

В самом деле, в соответствии с п. 4.5 гл. I в [2] имеются эпиморфизмы

$$
{ }^{\prime} E_{2}^{q 0} \rightarrow{ }^{\prime} E_{3}^{q 0} \rightarrow \cdots \rightarrow{ }^{\prime} E_{\infty}^{q 0}=\underset{r}{\lim }\left\{{ }^{\prime} E_{r}^{q 0}\right\}
$$

и включение

$$
{ }^{\prime} E_{\infty}^{q 0} \subset \mathbb{H}^{q}\left(\mathscr{C}^{*}\right)
$$

Кроме того (см. [2, гл. I, п. 4.5]), $\mathbb{H}^{q}\left(\mathscr{C}^{*}\right)$ эпиморфно отображается на

$$
{ }^{\prime \prime} E_{\infty}^{0 q} \subset \cdots \subset{ }^{\prime \prime} E_{r}^{0 q} \subset{ }^{\prime \prime} E_{r-1}^{0 q} \subset \cdots \subset{ }^{\prime \prime} E_{2}^{0 q}
$$

Композиция всех этих отображений и определяет $\alpha$.

ЗАмЕчАниЕ 4. Преобразование $\alpha$ определено в лемме, очевидно, для любого двойного комплекса (не обязательно построенного в связи с гиперкогомологиями), однородные составляющие которого равны нулю при $p<0$. Как преобразование $H^{q} F\left(\mathscr{C}^{*}\right) \rightarrow F H^{q}\left(\mathscr{C}^{*}\right)$ оно может быть определено и независимо от спектральных последовательностей. Если $\mathscr{Z}^{q}, \mathscr{B}^{q}$ - ядра и образы дифференциала в $\mathscr{C}^{*}$, то из точности $F$ слева имеем точную последовательность $0 \rightarrow F\left(\mathscr{B}^{q}\right) \rightarrow F\left(\mathscr{Z}^{q}\right) \rightarrow$ $F H^{q}\left(\mathscr{C}^{*}\right)$. В силу точности $F$ слева $F\left(\mathscr{Z}^{q}\right)$ - коциклы комплекса $F\left(\mathscr{C}^{*}\right)$. Кроме того, точность последовательности $0 \rightarrow F\left(\mathscr{Z}^{q-1}\right) \rightarrow F\left(\mathscr{C}^{q-1}\right) \rightarrow F\left(\mathscr{B}^{q}\right)$ с учетом точности $F$ слева обеспечивает включение в $F\left(\mathscr{B}^{q}\right)$ кограниц $F\left(\mathscr{C}^{*}\right)$, что и определяет отображение $H^{q} F\left(\mathscr{C}^{*}\right)$ в $F H^{q}\left(\mathscr{C}^{*}\right)$. Это отображение совпадает с $\alpha$ : в соответствии с общим описанием членов ${ }^{\prime} E_{2}^{q 0}$ (см. [2, гл. I, п. 4.2]) эти группы получаются именно факторизацией $F\left(\mathscr{Z}^{q}\right)$ по кограницам $F\left(\mathscr{C}^{*}\right)$, использованное же выше отображение $F\left(\mathscr{Z}^{q}\right) \rightarrow F H^{q}\left(\mathscr{C}^{*}\right)$ при аналогичной “расшифровке" $F H^{q}\left(\mathscr{C}^{*}\right)$ как члена " $E_{2}^{0 q}$ спектральной последовательности совпадает с фигурирующим в 
лемме. Определяемое таким образом преобразование $\alpha$ применялось для непосредственных (т.е. без спектральных последовательностей) доказательств формул типа Кюннета (точных последовательностей)

$$
0 \rightarrow F^{1} H^{n-1}\left(\mathscr{C}^{*}\right) \rightarrow H^{n} F\left(\mathscr{C}^{*}\right) \rightarrow F H^{n}\left(\mathscr{C}^{*}\right) \rightarrow 0
$$

и их обобщений вида

$$
0 \rightarrow F^{1}\left(\mathscr{Z}^{n-1}\right) \rightarrow H^{n} F\left(\mathscr{C}^{*}\right) \rightarrow F H^{n}\left(\mathscr{C}^{*}\right) \rightarrow F^{2}\left(\mathscr{Z}^{n-1}\right) \rightarrow 0
$$

(см. обзор подобного типа результатов в $[6$, гл. $8, \S 1]$ и обсуждения в связи с леммой 1.2 в [5]).

Отметим, что в интересуюшем нас конкретном случае изоморфизм

$$
{ }^{\prime} E_{2}^{p 0}=H_{-p}^{\varphi}(A ; \mathscr{G})={ }^{\prime \prime} E_{2}^{0 p}
$$

осуществляется как раз посредством $\alpha$, поскольку гомологии обратного предела комплексов (члены ' $E_{2}^{p 0}$ ) всегда отображаются в обратный предел гомологий этих комплексов (члены ${ }^{\prime \prime} E_{2}^{0 p}$ ).

Теорема была сведена выше к следующему утверждению.

ПРЕДЛОЖЕНИЕ 5. Из условия, что $\alpha:{ }^{\prime} E_{2}^{q 0} \rightarrow{ }^{\prime \prime} E_{2}^{0 q}-$ изоморфизмыь, при ${ }^{\prime \prime} E_{2}^{p q}=0$ для $p>0$ вытекает, ито ' $E_{2}^{q p}=0$ при $p>0$, и, следовательно, чmo $\mathbb{H}^{*}\left(\mathscr{C}^{*}\right)=H^{*} F\left(\mathscr{C}^{*}\right)$.

Заметим, что обрашение в нуль " $E_{2}^{p q}$ при $p>0$ еще не означает сходимость второй спектральной последовательности к гомологиям двойного комплекса, т.е. к $\mathbb{H}^{*}\left(\mathscr{C}^{*}\right)$ (см. пример 2.7 и пример f) из $\left.[8, \S 3]\right)$.

ДокАЗАТЕльство. Сначала фиксируем внимание на некотором отдельном члене ${ }^{\prime} E_{2}^{m 1}$. Пусть $\mathscr{C}^{*}(m)$ - комплекс, для которого $\mathscr{C}^{q}(m)=\mathscr{C}^{q}$ при $q \geqslant m$, $\mathscr{C}^{m-1}(m)=\mathscr{B}^{m}\left(\right.$ образ дифференциала $\left.\mathscr{C}^{m-1} \rightarrow \mathscr{C}^{m}\right)$ и $\mathscr{C}^{q}(m)=0$ при $q \leqslant m-2$. Очевидно наличие эпиморфизма $j(m): \mathscr{C}^{*} \rightarrow \mathscr{C}^{*}(m)$, тождественного при $q \geqslant m$ и совпадаюшего с дифференциалом в размерности $m-1$. Он порождает аналогичный эпиморфизм резольвент Картана-Эйленберга $\mathscr{J}^{*} \mathscr{C}^{*} \rightarrow \mathscr{J}^{*} \mathscr{C}^{*}(m)$ (вторая из которых естественным образом определяется первой), а поскольку $F$ сохраняет точность точных последовательностей, составленных $F$-ацикличными объектами, то $j(m)$ порождает также и эпиморфизм двойных комплексов $F\left(\mathscr{J}^{*} \mathscr{C}^{*}\right) \rightarrow$ $F\left(\mathscr{J}^{*} \mathscr{C}^{*}(m)\right)$, определяющих гиперкогомологии.

Заметим, что $H^{q}\left(\mathscr{C}^{*}(m)\right)=H^{q}\left(\mathscr{C}^{*}\right)$ при $q \geqslant m$ и $H^{q}\left(\mathscr{C}^{*}(m)\right)=0$ при $q<m$. Следовательно, " $E_{2}^{p q}(m)={ }^{\prime \prime} E_{2}^{p q}$ при $q \geqslant m$ и ${ }^{\prime \prime} E_{2}^{p q}(m)=0$ при $q<m$. Но для $\mathscr{C}^{*}(m)$ обе фильтрации в двойном комплексе регулярны, поэтому обе спектральные последовательности должны сходиться к $F H^{q}\left(\mathscr{C}^{*}(m)\right)={ }^{\prime \prime} E_{2}^{0 q}(m)$.

Рассмотрим поведение при $j(m)$ первой спектральной последовательности. Согласно [2, гл. I, п. 4.2]

$$
{ }^{\prime} E_{2}^{q p}=Z_{2}^{q p} /\left(Z_{1}^{q+1, p-1}+B_{1}^{q p}\right),
$$

где $Z_{r}^{q p}$ - элементы полной степени $q+p$ двойного комплекса $F\left(\mathscr{J}^{*} \mathscr{C}^{*}\right)$, принадлежащие $q$-му подкомплексу фильтрации по $q$, полный дифференциал от которых попадает в $(q+r)$-й подкомплекс фильтрации, а $B_{1}^{q p}$ - элементы степени $q+p$ из $q$-го подкомплекса фильтрации, служащие образами дифференциала 
из $(q-1)$-го подкомплекса. Из этого описания видно, что $j(m)$ индуцирует изоморфизмы ' $E_{2}^{q p} \rightarrow{ }^{\prime} E_{2}^{q p}(m)$ при $q \geqslant m$. В частности, ' $E_{2}^{q 0}=H^{q} F\left(\mathscr{C}^{*}\right)$ при $q \geqslant m$. Поскольку $\alpha$ коммутирует с отображениями комплексов, преобразования $\alpha:{ }^{\prime} E_{2}^{q 0}(m) \rightarrow{ }^{\prime \prime} E_{2}^{0 q}(m)$ при $q \geqslant m$ - изоморфизмы. Таким образом, первая спектральная последовательность двойного комплекса $F\left(\mathscr{J}^{*} \mathscr{C}^{*}(m)\right)$ должна сходиться к $F H^{q}\left(\mathscr{C}^{*}(m)\right)$, т.е. к $H^{q} F\left(\mathscr{C}^{*}(m)\right)={ }^{\prime} E_{2}^{q 0}(m)$ при $q \geqslant m$ и к нулевым группам при $q<m$.

В частности, поскольку необходимо, чтобы ${ }^{\prime} E_{2}^{q 0}(m)={ }^{\prime} E_{3}^{q 0}(m)$ при $q \geqslant m$, дифференциал $d_{2}:{ }^{\prime} E_{2}^{m 1}(m) \rightarrow{ }^{\prime} E_{2}^{m+2,0}(m)=H^{m+2} F \mathscr{C}^{*}(m)$ должен быть нулевьг. По построению комплекса $\mathscr{C}^{*}(m)$ имеем ${ }^{\prime} E_{2}^{m-2,0}(m)=0$. Это означает, что ${ }^{\prime} E_{2}^{m 1}(m)={ }^{\prime} E_{3}^{m 1}(m)=\cdots={ }^{\prime} E_{\infty}^{m 1}(m)$. Поскольку спектральная последовательность при $q \geqslant m$ сходится к $H^{q} F\left(\mathscr{C}^{*}\right)$, то должно быть ' $E_{\infty}^{m 1}(m)=0$. Следовательно, ${ }^{\prime} E_{2}^{m 1}={ }^{\prime} E_{2}^{m 1}(m)=0$.

Таким образом, ' $E_{2}^{q 1}=0$ для всех $q$. Ясно, однако, что такие же аргументы применимы к членам ${ }^{\prime} E_{2}^{q 2},{ }^{\prime} E_{2}^{q 3}, \ldots$. Тем самым, ${ }^{\prime} E_{2}^{q p}=0$ при $p>0$. Предложение доказано.

Поскольку в изучаемой ситуации " $E_{2}^{p q}=\cdots={ }^{\prime \prime} E_{r}^{p q}=\cdots$, при этом всегда " $E_{\infty}^{p q} \subset{ }^{\prime \prime} E_{r}^{p q}$, очевидным следствием проведенных рассуждений является сходимость к $\mathbb{H}^{*}\left(\mathscr{C}^{*}\right)=H^{*} F\left(\mathscr{C}^{*}\right)$ и второй спектральной последовательности (в том числе равенство " $\left.E_{\infty}^{p q}={ }^{\prime \prime} E_{2}^{p q}\right)$. Как правило, вторая спектральная последовательность сходится к упоминавшимся выше “сильным" когомологиям двойного комплекса, в том числе в ситуациях, когда они отличаются от обычных (пример 2.7 и пример f) из $[8, \S 3])$. Одним из признаков такой сходимости второй спектральной последовательности (применимым как раз в нашем случае) является обрашение в нуль при некотором $r$ членов " $E_{r}^{p q}$ для $p>p_{0}$ (более общие условия даны в теореме 1.4 и следствии 1.5 в [8]). В сходимости к сильным когомологиям можно убедиться и непосредственно. Они определяются (рассматриваемьм в полной градуировке) комплексом

$$
N^{*}=\lim _{m \rightarrow-\infty}\left\{F\left(\mathscr{J}^{*} \mathscr{C}^{*}(m)\right)\right\}
$$

для когомологий которого имеют место известные точные последовательности (вариант соотношений Кюннета)

$0 \rightarrow \lim _{m \rightarrow-\infty}{ }^{1}\left\{H^{n-1} F\left(\mathscr{J}^{*} \mathscr{C}^{*}(m)\right)\right\} \rightarrow H^{n}\left(N^{*}\right) \rightarrow \lim _{m \rightarrow-\infty}\left\{H^{n} F\left(\mathscr{J}^{*} \mathscr{C}^{*}(m)\right)\right\} \rightarrow 0$.

Поскольку при $m<n$ групшы $H^{n} F\left(\mathscr{J}^{*} \mathscr{C}^{*}(m)\right)$ не зависят от $m$ и равны $H^{n} F\left(\mathscr{C}^{*}\right)$, функторы $\lim ^{1}$ обращаются в нуль и $H^{n}\left(N^{*}\right)=H^{n} F\left(\mathscr{C}^{*}\right)={ }^{\prime \prime} E_{2}^{0 q}$. В частности, в изучаемой ситуации сильные когомологии двойного комплекса совпадают с обычньми. Отметим, что это совпадение, по-видимому, не является следствием довольно общих условий изоморфизма обычных и сильных гиперкогомологий, представленных в теореме 2.9 в [8].

В заключение замечание о гомологиях окружения для рассматриваемого $A \subset X$. Нетрудно убедиться, что система $\left\{H_{n}^{\varphi}(W ; \mathscr{G})\right\}$ почти постоянна тогда и только 
тогда, когда таковой оказывается проективная система $\left\{H_{n}^{\varphi}(W \backslash A ; \mathscr{G})\right\}[7$, лем-

ма 3.2]. Было бы естественно, очевидно, постоянную часть $H_{n}^{\varphi}(\langle\langle A\rangle ;$; $)$ системы $\left\{H_{n}^{\varphi}(W \backslash A ; \mathscr{G})\right\}$ считать (по определению) гомологиями окружения $A$ в $X$. Однако для того, чтобы установить их изоморфизм группам $H_{n+1}^{\varphi}(A: X \backslash A ; \mathscr{G})$, необходима интерпретация $\left.H_{n}^{\varphi}(\langle A\rangle\rangle ; \mathscr{G}\right)$ как гипергомологий системы $\left\{H_{n}^{\varphi}(W \backslash A ; \mathscr{G})\right\}$ для функтора $\lim$. Такая интерпретация постоянной части проективной системы $\left\{H_{n}^{\varphi}(W \backslash A ; \mathscr{G})\right\}$ может рассматриваться в качестве одного из следствий работы.

\section{Список литературы}

1. Картан А., Эйленберг С. Гомологическая алгебра. М.: ИЛ, 1960.

2. Годеман Р. Алгебраическая топология и теория пучков. М.: ИЛ, 1961.

3. Jensen C. U. Les foncteours dérivés de lim et leurs applications en théorie des modules // Lect. Notes in Math. V. 254, 1972.

4. Кузьминов В. И. Производные функторы проективного предела и классы расширений // Сиб. матем. журн. 1971. Т. 12. № 2. С. 384-396.

5. Скляренко Е. Г. Некоторые применения функтора $\lim ^{1} / /$ Матем. сб. 1984. Т. 123. № 3 . C. 369-390.

6. Сћляренко Е. Г. Гомологии и когомологии общих пространств // Итоги науки и техники. Соврем. пробл. матем. Фундам. направл. Т. 50. М.: ВИНИТИ, 1989. С. 129-266.

7. Скляренко Е. Г. Гомологии и когомологии связи между множествами. Гомологии и когомологии окружения замкнутого множества // Изв. РАН. Сер. матем. 1992. Т. 56. № 5 . C. $1040-1071$.

8. Скляренко Е.Г. Гипер(ко)гомологии для точных слева ковариантных функторов и теория гомологий топологических пространств // УМН. 1995. Т. 50. № 3. С. 109-146.

Московский государственный

Поступила в редакцию

университет им. М.В. Ломоносова

09.01 .1996 